\title{
Curbing the Cost of Cancer Treatment- A Pipe Dream or a viable possibility for the
} Government?

Sharan $\mathrm{K}^{1}$

${ }^{1}$ Associate Professor, Department of Radiotherapy, Shirdi Saibaba Cancer Hospital, Manipal, Karnataka,

India.

\section{Editorial}

\section{Corresponding Author: \\ Dr. Krishna Sharan MD, \\ Associate Professor, \\ Department of Radiotherapy, \\ Shirdi Saibaba Cancer Hospital, \\ Manipal, Karnataka, India. \\ Email: tk.sharan@gmail.com}

The word 'cancer' evokes strong feelings of despair and helplessness in most people. This is not surprising, since cancer has been known and recognized as a deadly disease for ages, and the stigma associated with the word can be equalled only by very few other ailments. According to the Globocan 2008 report by the International Agency for Research in Cancer (IARC), 27,800 patients were diagnosed with cancer, while around 20,000 died due to cancer in Nepal in the year $2008^{1}$. It is, however, a misconception that all cancers are incurable, and that the patient with cancer is bound to die with the disease shortly.

Cancers are usually treated with a multimodal approach, principally including surgery, use of ionizing radiation and use of injectable or oral medicines. Tremendous amount of research is going on in cancer, and the resultant progress has seen increasing cure rates with decreasing rates of side effects in many cancers. Cancers like lymphoma and germ cell tumors that were uniformly fatal few decades ago can now be cured in most of the patients.

However, with advances in treatment, the improved cure rates have almost always been accompanied with rising costs. The cost of modern therapy has spiralled out of the reach of many a lower and middle socioeconomic class patient. Annual direct costs for cancer care in the United States are projected to rise- from \$104 billion in 2006 to over \$173 billion in 2020 and beyond, and the 'rising cost' situation in the rest of the world can be expected to be no different ${ }^{2}$. This increase has been driven by a dramatic rise in both the cost of therapy and the extent of care.

In their excellent article "Bending the cost curve in cancer care", published in the New England Journal of Medicine, Smith et al noted that in the United States, the sales of anticancer drugs are now second only to those of drugs for heart disease, and $70 \%$ of these sales come from products introduced in the past 10 years. Moreover, the medicines are exorbitantly priced, and in many cases, the costeffectiveness ratios far exceed commonly accepted thresholds ${ }^{3}$. The article went on to suggest changing a few practices in the health care system that could reduce the burden of cancer treatment. This is of lesser relevance to the Nepalese health system, principally because in most instances the expenses of treatment are usually borne by the patient himself, rather than a medical insurance company or the Government.

The concept of controlling cancer in a developing country is still relatively new, making it difficult to estimate the costs and cost-effectiveness of various prevention and treatment strategies. Some of the strategies have been discussed by various organizations, including the World Health Organization (WHO) regarding control of cancer in developing countries. A few of these have been compiled and are discussed below. There appears to be no 'fit-for-all' solution. Study of health care technologies and health service in each country, along with cost evaluations, will shed more light on strategies which are likely to benefit that country. Pilot programs are an ideal way to begin, according to the Disease Control Priorities Project (DCPP) ${ }^{4}$.

\section{Primary Prevention}

Primary prevention aims to reduce or eliminate exposure to cancer-causing risk factors. Primary prevention is in many 
instances the best strategy in controlling diseases, cancer included. Primary prevention constitutes of preventive strategies like practicing a healthy life style with good physical exercise, avoiding tobacco and drugs/habit forming substances, and of prophylaxis strategies like immunization. According to the Globocan 2008, cervical cancer contributes to nearly $13 \%$ of all cancers diagnosed annually in Nepal, and to $21 \%$ of cancers diagnosed among women. Vaccination with anti-HPV vaccines has been estimated to bring down the cervical cancer rates by as much as $70 \%$, and this strategy should be seriously considered by the Government. Similarly, a robust national tobacco and alcohol consumption programs have the potential to bring down the incidence of lung, gastrointestinal and several other cancers significantly.

\section{Secondary Prevention}

Secondary prevention involves early detection of cancers, leading to higher cure rates and consequent lesser economic burden in terms of life years. Screening and early detection is more meaningful for cancers that are of a significant burden in the society, and can be cured in most patients if treated at an early stage. Thus, though lung cancer is a significant burden, lack of good screening in addition to poorer results of treatment even if detected early, does not have much scope for control with secondary prevention. On the other hand, cervical cancer usually affects women at a time of life when they are critical to social and economic stability. A publication by Goldie et.al., assessing the cost-effectiveness of screening for cervical cancers in developing countries, suggested an estimated 30$36 \%$ reduction in lifetime risk of cervical cancer with a single screening (performed at the age of 35 years) using HPV DNA testing ${ }^{5}$.

\section{Cancer treatment}

Cancer treatment principally involves 3 modalities- surgical, radiation and medical oncology. While surgical oncology, with provision of good basic facilities and well trained staff, can be of less economic burden, radiotherapy and chemotherapy usually carry significant expenses. Radiotherapy set-up involves a very expensive infrastructure, in addition to availability of well-trained staff, maintenance and quality assurance of equipment, etc. Bringing down the cost of radiotherapy to the patient requires careful planning at a national level. The regional requirements should be assessed and treatment centers must be planned accordingly. Cobalt- 60 teletherapy units are far cheaper to run and maintain than a linear accelerator. While they are definitely not the best available, they can provide effective treatment at much lesser cost.
More sophisticated and specialized technologies can be more centralized, and a good network and referral system should be developed, so that patients requiring complex treatment are not denied. Finally, chemotherapy forms a very important component of treatment for cancer. While surgery and radiotherapy can be used to tackle disease of limited volumes, some form of systemic treatment is the only option for controlling disseminated disease. Modern chemotherapy has become very expensive, and this is attributed to several reasons. It is probably very difficult to cut on the expense of research and development. However, other factors which contribute to high prices include corruption, and excess profit margins. The whole purpose of this article was the realization that there is an insane margin of profit at every intermediate step in the process of drug manufacture to the patient purchasing it from the retail shop. Some sort of action has to be taken to curb this, or else modern chemotherapy is soon going to be out of the reach of most people. Two important strategies that can be adopted to control these aspects include:

\section{Price regulation}

Price regulation implies that the retail price of a drug should bear some logical relation to its manufacturing cost. The real manufacturing cost is often a very small fraction of the retail price. The consumers at the retail counter pay many times more than the price at which the drugs are provided to the traders or to the government.

There are potential problems involved with price regulation; limiting profit can result in fewer manufacturers, shortage of medicines, lower quality medicines and opportunities for spurious drug manufacturers, etc. Despite this, some sort of a middle path has to be identified by the governments, so that the average consumer is able to afford the treatment

\section{Reducing corruption}

Corruption in the pharmaceutical sector occurs throughout all stages of the medicines chain, from research and development to dispensing and promotion. Unethical practices along the chain can take many forms such as falsification of evidence, mismanagement of conflict of interest, or bribery. The large number of steps in the medicines chain allows numerous opportunities for unethical practices to take place. This in consequence leads to more expensive medicines, reduced availability and opportunities for sale of spurious drugs.

To reduce the menace of corruption in medicines, WHO launched the Good Governance for Medicines (GGM) program in $2004^{6}$. By applying transparent, accountable administrative procedures and by promoting ethical 
practices, GGM provides support for countries to curb corruption. The program assists countries through a threestep process of assessing their vulnerabilities to corruption, and developing and implementing specific programs to maintain efficient health-care systems that are not undermined by the abuse of corruption.

\section{Conclusion}

Cancer is on the rise, and is known to cause significant impact on the health and socio-economics of a Nation. Initiatives must be taken by the Government to bring down the preventable cancers by primary and secondary prevention strategies. Steps must also be taken to regulate the prices of anti-cancer medicines, and corruption must be checked. Only then will it be possible to provide affordable treatment for a patient suffering from cancer. Lastly, despite the phenomenal growth of Oncology as a science, the single most important factor that has improved the outcome of a patient with cancer is awareness. It is the knowledge of the lady who just felt a lump in her breast that she needs to show it to a doctor, or the understanding of a person that practicing a healthy lifestyle significantly reduces the risk of developing cancer, that has made the maximum impact on the outcome of this disease called cancer. A simple step of spreading awareness in the hazards of smoking and tobacco consumption and conducting smoking cessation programs could bring down the incidence of various cancers like lung cancers, head and neck cancer, gastrointestinal cancers, etc. significantly.

\section{Conflict of Interests}

The author has no conflict of interest arising from the study.

\section{References}

1. Globocan 2008: Cancer incidence, Mortality and Prevalance in 2008 [online] 2008 [cited 2011 November 27]. Available from: URL: http://globocan.iarc.fr/

2. Mariotto $A B$, Yabroff KR, Shao Y, Feuer EJ, Brown ML. Projections of the cost of cancer care in the United States: 2010-2020. J Natl Cancer Inst 2011;103:117-28.

3. Hillner BE, Smith TJ. Efficacy does not necessarily translate to cost effectiveness: a case study in the challenges associated with 21 st-century cancer drug pricing. J Clin Oncol 2009;27:2111-3.

4. Controlling Cancer in Developing Countries: Prevention and treatment strategies merit further studies [online] 2007 [cited 2011 November 27] Available from: URL: http://www.dcp2.org/file/79/DCPP-Cancer.pdf

5. Cost-Effectiveness of Cervical-Cancer Screening in Five Developing Countries Sue J. Goldie, M.D. N Engl J Med 2005;353:2158-68.
6. Good governance for medicines programme, World Health Organization [online] 2010 [cited 2011 November 27] Available from: URL: http://www.who.int/medicines/ ggm/en/index.html

\begin{tabular}{|l|l|}
\hline \multicolumn{2}{|c|}{ Article Information } \\
\hline \multicolumn{2}{|c|}{ Article history } \\
\hline Received & 10 February 2012 \\
Received in revised form & 15 March 2012 \\
Accepted & 20 March 2012 \\
\hline
\end{tabular}

